\title{
Maximización del Throughput en una red de radio cognitiva basado en la probabilidad de falsa alarma
}

\author{
Maximizing Throughput in a radio network based on cognitive \\ probability of false alarm
}

Ricardo Alonso Ferro Bolívar

Ingeniero electrónico, estudiante de la Maestría en Ciencias de Información y las Comunicaciones de la Universidad Distrital Francisco José de Caldas. Bogotá, Colombia.rifer2411@gmail.com

\section{Luis F. Pedraza}

Ingeniero electrónico, magíster en Ciencias de la Información y las Comunicaciones. Docente e investigador de la Universidad Distrital Francisco José de Caldas. Bogotá, Colombia.lfpedrazam@udistrital.edu.co

\section{CÉsar Hernández}

Ingeniero electrónico, magíster en Ciencias de la Información y las Comunicaciones. Docente e investigador de la Universidad Distrital Francisco José de Caldas. Bogotá, Colombia.cahernandezs@udistrital.edu.co

Clasificación del artículo: Investigación (Conciencias)

Fecha de recepción: 3 de junio de 2011

Fecha de aceptación: 29 de agosto de 2011

Palabras clave: Espectro electromagnético, probabilidad de falsa alarma, radio cognitiva, throughput.

Key words: Electromagnetic spectrum, probability of false alarm, radio cognitive, throughput.

\section{RESUMEN}

En este artículo se muestra los problemas que existen en el enrutamiento de redes en la radio cognitiva, teniendo en cuenta un parámetro muy importante como es el comportamiento del throughput. De esta manera, se plantea un escenario para una red Ad Hoc tipo inalámbrica con características de radio cognitiva, implementando protocolos de enrutamiento como Weighted Cumulative Expected Transmission Time (WCETT) y algunos protocolos de enlace Mac. El objetivo principal de este estudio radica en implementar un algoritmo de optimización para maximizar el throughput manejando 


\section{con-ciencias}

un parámetro de control, como es la probabilidad de falsa alarma. En el desarrollo de este trabajo se mostrará el modelamiento de un algoritmo de optimización que maximice el throughput en el momento que se efectúe el sensado del espectro en un entorno de radio cognitiva sin que exista cooperación en el sensado. Y de esta forma poder demostrar gráficamente el comportamiento que tiene el throughput en un entorno cognitivo analizando un parámetro de control y optimización, como la probabilidad de falsa alarma.

\section{ABSTRACT}

This paper shows the problems that exist in the routing of cognitive radio networks taking into ac- count a very important parameter as the behavior of throughput. In this way, a scenario for a Wireless Ad Hoc Network with features of cognitive radio, implementing routing protocols such as Time Weighted Cumulative Expected Transmission (WCETT) and some handshakes Mac, the main objective of this study is to implement an algorithm optimization to maximize throughput handled as a control parameter is the probability of false alarm. In developing this paper will show the modeling of an optimization algorithm that maximizes throughput at the moment is made of the spectrum sensing in cognitive radio environment with no cooperation in the sensing. And in this way to graphically demonstrate the behavior that the throughput in a cognitive analysis of a parameter optimization and control as the probability of false alarm.

\section{INTRODUCCIÓN}

En los últimos años hemos sido testigos de un efusivo aumento en la demanda de la utilización de las bandas de frecuencia. El principal problema radica en que las políticas actuales de asignación de bandas frecuenciales son demasiado estrictas y no permiten un uso óptimo del espectro radioeléctrico disponible. Así mismo, es frecuente encontrar bandas del espectro altamente congestionadas como la telefonía celular y a su vez hallar bandas subyacentes subutilizadas. De esta forma, se crea un paradigma en los sistemas de comunicaciones, el cual es mejorar el uso de las bandas de frecuencias.

Así nacen las tecnologías de radio cognitiva, las cuales permiten a los usuarios acceder a los llamados huecos espectrales, sin interferir perjudicialmente en las bandas licenciadas. La radio cognitiva es considerada como la tecnología esencial para resolver los problemas de escasez del espectro [1].
En esta investigación se realiza un enfoque sobre el principio cognitivo en la lógica de una red inalámbrica en lo que respecta al enrutamiento. Para este objetivo, se revisa en primer lugar las investigaciones existentes sobre la aplicación de las redes cognitivas y el problema de enrutamiento [2].

\section{ESTADO DEL ARTE}

Dentro del marco conceptual se exponen un par de investigaciones cuyo objetivo es plantear una serie de métodos para poder maximizar un parámetro como el throughput en el enrutamiento de redes de radio cognitiva.

\subsection{Maximización del throughput en redes de radio cognitiva}

Dentro de los estudios realizados para maximizar el throughput en las redes de radio cognitiva. Se ha propuesto un estudio en el que se evalúan dos escenarios. El primero es cuando existe maximi- 


\section{con-ciencias}

zación del throughput sin cooperación en el sensado del espectro y el otro es cuando sí existe cooperación. Con base en estos estudios existen dos parámetros relacionados al sensado del espectro los cuales son la probabilidad de detección y la probabilidad de falsa alarma.

Investigaciones previas han demostrado que el desempeño en el sensado del espectro es altamente dependiente del espacio que es asignado para este. El objetivo de la relación sensado-throughput es poder alcanzar una tasa de bit alcanzable para garantizar una calidad de servicio adecuada. Por tanto, para alcanzar el límite superior, los símbolos de distribución gaussiana se asumen para ser transmitidos. Sin embargo, en la práctica los bits de datos modificados son siempre modulados hacia los símbolos extraídos de una constelación dada y luego estos símbolos son delimitados a tramas y transmitidos a través de canales inalámbricos hacia su destino. Esta investigación plantea un algoritmo para maximizar el throughput, aplicando enrutamiento de una red de radio cognitiva, en este caso, sin cooperación en el sensado del espectro [3], [4].

\subsection{Maximización del throughput en redes de radio cognitiva con limitación de usuarios primarios}

En estas notas se considera una red de radio cognitiva punto a multipunto, que acciona una serie de canales con una red primaria. Dentro de la red de radio cognitiva una estación base (BS) controla y soporta un conjunto de suscriptores de servicios inalámbricos fijos.

El objetivo de esta investigación es maximizar el throughput del enlace de bajada en las redes de radio cognitiva sin afectar el desempeño de los usuarios primarios. Para eso se propone una mezcla de algoritmos de control que requieren una interacción mínima entre los dispositivos primarios y cognitivos. Primero, una fuente distribuida de procesos actualizados es empleada en los nodos primarios y cognitivos para maximizar la cobertura de las redes cognitivas, mientras se mantenga baja la relación señal a ruido de los transmisores primarios. Después de eso la asignación del canal se lleva a cabo dentro de la red cognitiva para maximizar el throughput del enlace descendente. Los resultados numéricos se obtienen del comportamiento y rendimiento de algunos algoritmos propuestos entre los cuales se encuentran:

\subsubsection{MDCA (Algoritmo de control para una mezcla distribuida y centralizada)}

Posee una fase de control y otra fase de asignación de canal. En la primera fase, para cada canal se halla la potencia de transmisión máxima de cada estación base, junto con la potencia de transmisión de los transmisores primarios (PTXs), de tal modo que las limitaciones de señal a ruido de todos los receptores primarios (PRXs) se cumplan.

Por otro lado, como la fase 2 solo involucra BS y equipo local del cliente (CPEs), un esquema de asignación de canal centralizado es apropiado.

\subsubsection{NCSM (Algoritmo de enlace simple sin cooperación)}

La no cooperación quiere decir que durante el proceso de control en la potencia en la fase 1. Los PTXs no ajustan su potencia de transmisión junto con las estaciones base. En cambio en este algoritmo NCSM cada PTX fija la potencia de transmisión al valor máximo. Luego la estación base (BS) intenta maximizar esta potencia de transmisión sobre cada canal, sujeta a las limitaciones de señal a ruido de todos los canales PRXs

\subsubsection{NCMM (Algoritmo deenlace máximo sin cooperación)}

La fase 1 de este esquema NCMM es similar al esquema NCSM descrito anteriormente, allí no 


\section{con-ciencias}

hay cooperación de los transmisores primarios. Por otro lado, en la fase 2, el enlace bilateral máximo es empleado para optimizar el canal asignado. Para comparar el desempeño entre MDCA y NCMM se puede ver el impacto que tiene la cooperación de los usuarios primarios en la fase $1[5,6]$.

\section{METODOLOGÍA}

Para el modelamiento de nuestro sistema se va a establecer una relación con la probabilidad de falsa alarma y el algoritmo de optimización planteado para un escenario de radio cognitiva cuando no existe cooperación en el sensado del espectro electromagnético. Dentro de las variables por considerar encontramos:

Trama de datos $=$ Duración $\boldsymbol{T}$

Sensado del periodo $=$ Extensión $\tau$

Periodo de transmisión $=T-\tau$

$H 0=$ Ausencia de Usuarios Primarios (PU)

$H 1=$ Presencia de $P U$

Frecuencia de muestreo $=\boldsymbol{f} s$

Probabilidad de falsa alarma $=P_{f(\dot{o}, \tau)}$

Así mismo, la probabilidad de falsa alarma puede ser expresada de la siguiente forma:

$$
P_{f(\grave{o}, \tau)}=\left((\grave{o}-1) * \sqrt{\frac{\tau f_{s}}{2}}\right)
$$

Si el sensado del espectro indica que el enlace PU está activo, para evitar interferencias los usuarios CR guardaran silencio [7].

Si el sensado deduce que el enlace PU está inactivo, inmediatamente una secuencia de los símbolos modulados de la forma BPSK con velocidad de 1bit/símbolo serán transmitidos.
Símbolos modulados $=L$ (duración)

Entonces para una duración $T$

$N=T / L$ simbolos

Cuando los usuarios CR transmiten información puede existir la posibilidad de que haya ausencia de usuarios primarios. Así mismo se puede implementar la probabilidad de falsa alarma, de la siguiente forma:

$1-P_{0}:$ Probabilidad que los símbolos transmitidos se reciban correctamente

$P_{0}=B E R$

Si los usuarios de radio cognitiva transmiten con una SNR de $\rho_{s}$ el BER puede ser expresado así:

$$
P_{0}=Q\left(\sqrt{\rho_{s}}\right)
$$

De esta manera una duración con medida $\tau$ es usada para sensar el espectro [4].

$$
[(T-\tau) / L]_{\begin{array}{l}
\text { Simbolos pueden ser transmitidos } \\
\text { en trama }
\end{array}}
$$

Y los símbolos nulos se denotan como: $\tau_{N}$

Como resultado el throughput para este caso se denota:

$$
\begin{gathered}
T\left(H_{0}\right)=\left(1-\frac{\tau+\tau_{N}}{T}\right)\left(1-P_{0}\right)^{\left[\frac{T-\tau}{L}\right]} \\
T\left(H_{0}\right) \mathrm{P}\left(H_{0}\right)\left(1-P_{f}(\grave{o}, \tau)\right) \\
T_{0}(\grave{o}, \tau)=\mathrm{P}\left(H_{0}\right)\left(1-P_{f}(\grave{o}, \tau)\right) T\left(H_{0}\right) \\
T_{0}(\grave{o}, \tau)=\mathrm{P}\left(H_{0}\right)\left(1-P_{f}(\grave{o}, \tau)\right) \\
\left(1-\frac{\tau+\tau_{N}}{T}\right)\left(1-P_{0}\right)^{\left[\frac{T-\tau}{L}\right]}
\end{gathered}
$$




\section{con-ciencias}

Después de realizado el planteamiento matemático se considera el throughput como $\mathrm{T}_{0}(\mathrm{n})$, la cual es una función unimodal de argumento $\mathbf{x}$ continuo, y que es la alternativa continua de $\mathbf{n}$.

Una función unimodal se incrementa linealmente hasta que alcanza su valor pico y rápidamente decrece $T_{0}(n)$. Puede ser una función unimodal si cumple las siguientes condiciones [8]:

$$
\begin{aligned}
& \frac{d T_{0}(x)}{d x(0)}>0 \\
& \frac{d T_{0}(x)}{d x(N)}<0
\end{aligned}
$$

Con base en este teorema se ha planteado un algoritmo de optimización del throughput:

a.) Inicializa en $\boldsymbol{n}=\boldsymbol{1}$

b.) calcula $T_{0}(n+1)$ y compara $T_{0}(n)$ con $T_{0}(n+1)$

c.) Si $T_{0}(n+1)>T_{0}(n)$ para $\boldsymbol{n}=\boldsymbol{n}+1$ salta al paso 2

d.) Fin de la búsqueda $\boldsymbol{n}$ es la solución óptima correspondiente al valor máximo de throughput [4].

\section{RESULTADOS}

Para desarrollar las respectivas simulaciones se ha utilizado el software NS 2.31 sobre la plataforma Ubuntu. De esta forma se ha planteado un escenario para redes $A d H o c$, con un entorno basado en el enrutamiento de radio cognitiva. La configuración del escenario está planteada para una red inalámbrica tipo wireless, manejando tráfico $c b r$ e implementando los siguientes parámetros de simulación (Tabla 1).

Dentro del entorno cognitivo, se plantean 11 nodos, de los cuales 7 son usuario CR y 4 usuarios primarios, los cuales están en color rojo. El
Tabla 1. Parámetros de simulación.

\begin{tabular}{|l|l|}
\hline ATRIBUTO & VALOR \\
\hline Tipo de antena & Omnidireccional \\
\hline Protocolo de routing & Aodv \\
\hline Paquetes Max. en cola & 50 \\
\hline Tipo Mac & Macng \\
\hline № nodos & 11 \\
\hline № canales/radio & 5 \\
\hline Tipología & Random \\
\hline Tiempo de simulación & 50 \\
\hline
\end{tabular}

entorno de simulación bajo NS2 se muestra en las Fig. 1 y 2 , la cual es generada a través de un archivo .nam:

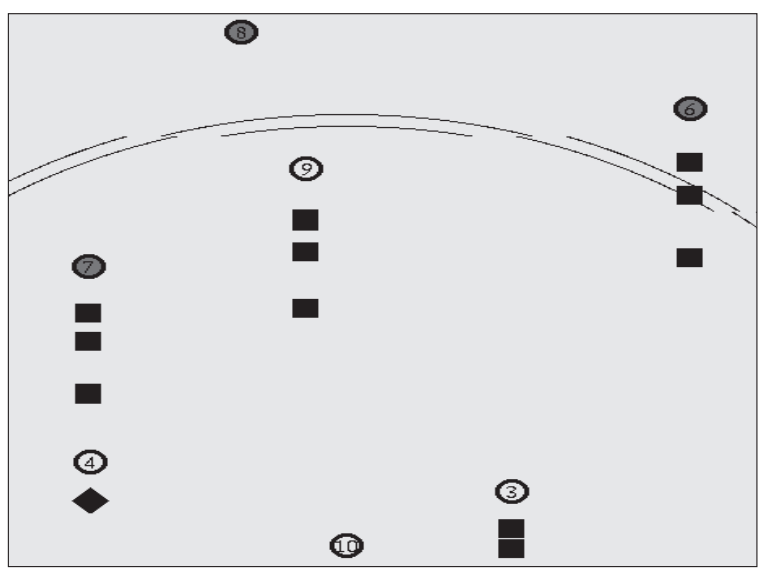

Fig. 1. Throughput con Pf de 0, 4. Vista 1 escenario de simulación.

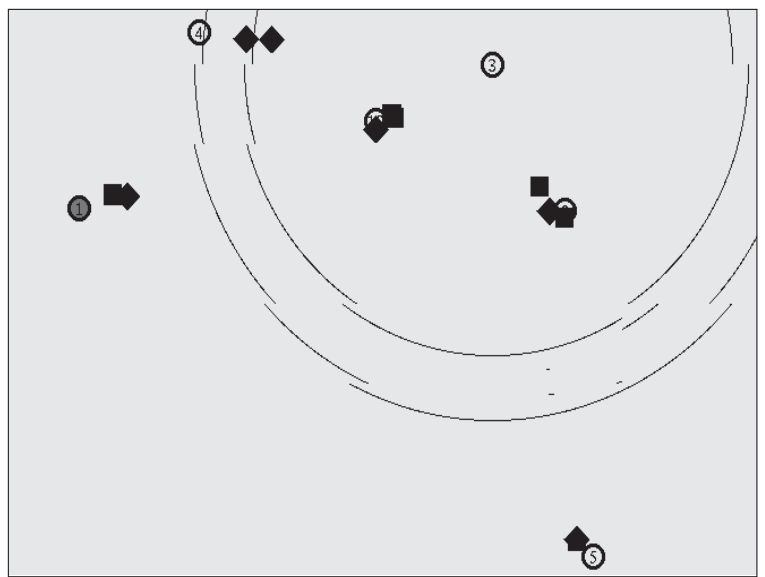

Fig. 2. Vista 2 escenario de simulación. 
En el análisis desplegado a partir del desarrollo y modelamiento se plantea tres entornos de configuración del escenario. En dos de los cuales se implementa el parámetro de probabilidad de falsa alarma.

En el primer entorno de configuración no se aplica ningún parámetro de control respecto al throughput. De tal forma, el procedimiento de transmisión entre cada nodo posee un comportamiento aleatorio.

En la Fig. 3 se observa cómo es el comportamiento del throughput sin aplicar ningún parámetro de control.

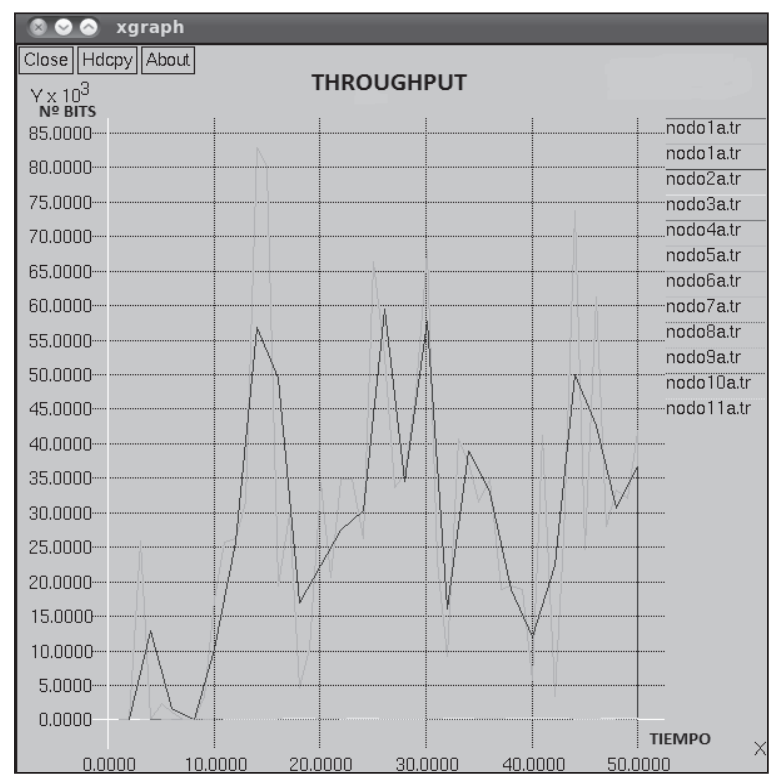

Fig. 3. Throughput con Pf de 0, 4.

En el segundo entorno de configuración se aplica una $P_{f}$ con un valor de 0,8 y se grafica el comportamiento del throughput en función del tiempo independiente para cada nodo. Se muestra en la Fig. 4.

En la Fig. 5 se muestra el comportamiento del throughput con una probabilidad de falsa alarma de 0,4 . En la cual se alcanzan valores de aprox. $150 \times 10^{6}$ bits por unidad de tiempo.

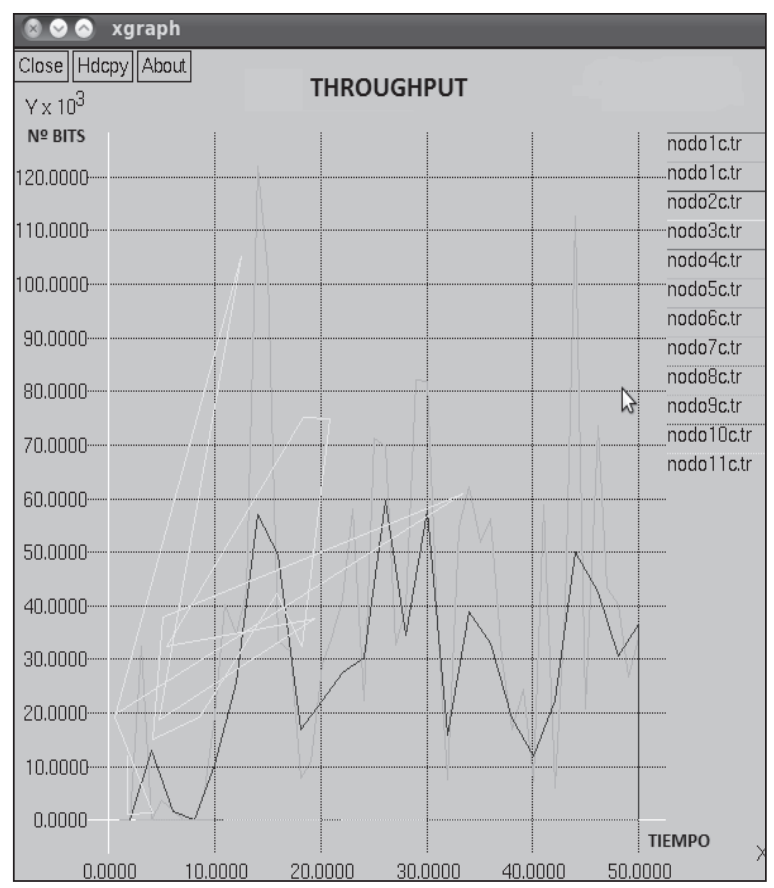

Fig. 4. Throughput con Pf de 0, 8.

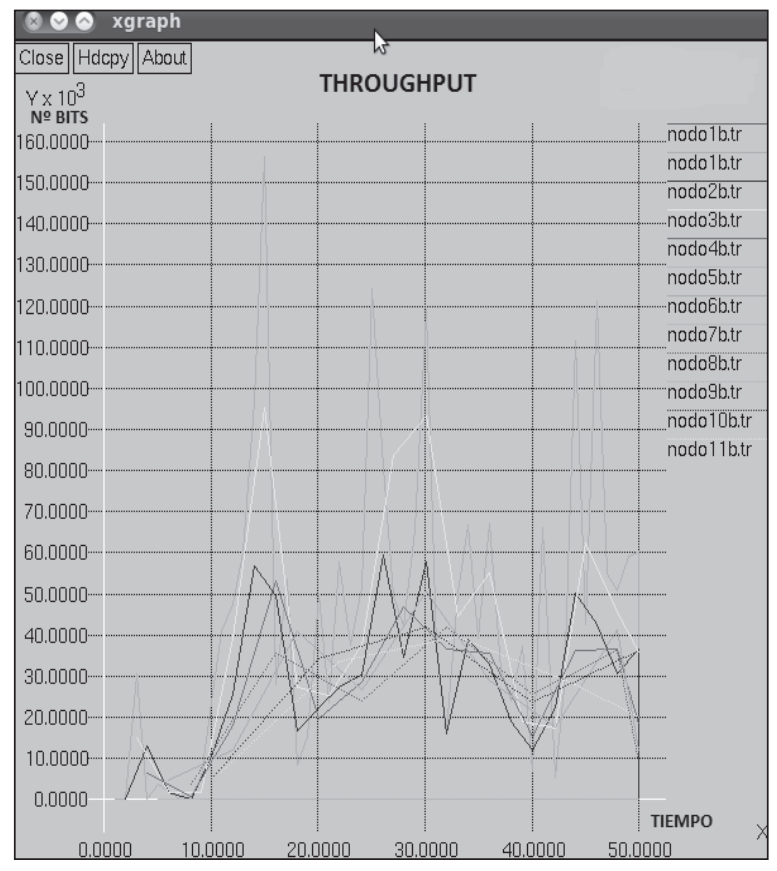

Fig. 5. Throughput con Pf de 0, 4.

En la Fig. 6 se muestra el valor medio de cada uno de los escenarios planteados con los valores de throughput para cada nodo. 


\section{con-ciencias}

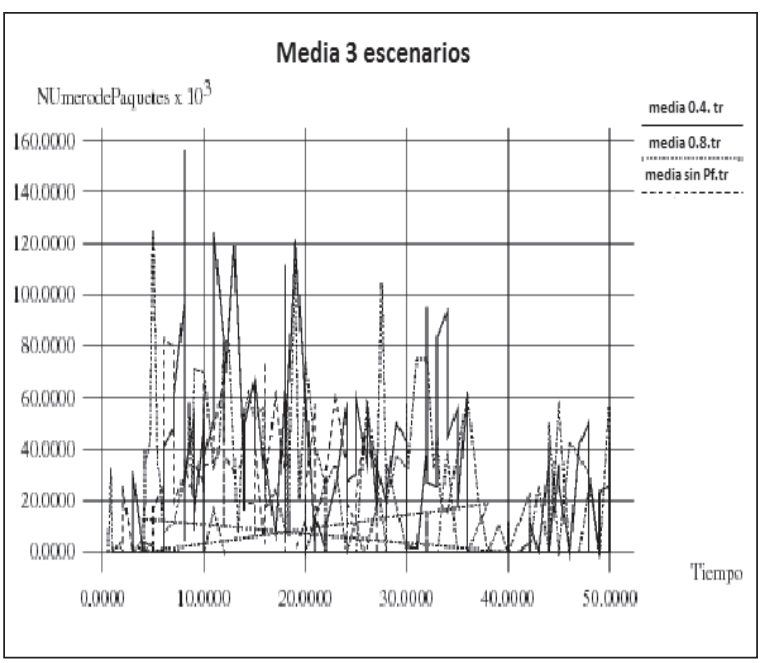

Fig. 6. La media del throughput en los 3 escenarios.

\section{CONCLUSIONES}

La baja probabilidad de falsa alarma generará una mejor posibilidad para la transmisión de usuario CR y de esta forma un incremento en el throughput.

Con una probabilidad de falsa alarma de 0.4 se alcanza niveles de maximización en el throughput de aproximadamente el $30 \%$ respecto a una probabilidad de 0.8 .

Si no se aplica el parámetro de probabilidad de falsa alarma no se puede apreciar un mecanismo de control para la optimización del throughput.

\section{REFERENCIAS}

[1] S. Choe, "Throughput, delay, and packet capture effects in rayleigh fading of a cognitive radio packet network," Department of Information, Communication, and Electronic Engineering the Catholic University of Korea, Bucheon, 2009.

[2] Y. Xia and F. Hu, Cognitive Radio Networks, (CRC). Taylor \& Francis Group, an information business by Taylor and Francis Group LLC, 2009.

[3] E. Chu, Y. Liang, Y. Liang and Y. Zeng. Optimization of Cooperative Sensing in Cognitive Radio Networks: A SensingThroughput Tradeoff View, IEEE Transactions on Vehicular Technology, vol.58, no. 9, pp. 5294-5299, Nov. 2009.

[4] Q. Wang, D. Wu and Y. Wang. "Throughput maximization in cognitive radio networks," National Mobile Communications Research Laboratory Southeast University, Nanjing China, 2009.
[5] A. Hoang, Y. Liang and MdHabibul Islam. "Maximizing throughput of cognitive radio networks with limited primary user's cooperation," IEEE International Conference on Communications, Singapore, 2008.

[6] Y. Liang, Y. Zeng, E. Peh, and A. Hoang, Sensing-Throughput Tradeoff for Cognitive Radio Networks, IEEE Transactions Wireless Communications, vol. 7, no. 4, pp. 1326-1337, Apr. 2008.

[7] S. Zheng, Y. Liang, P. Yuen and A. Hoang, "Cross-layered design of spectrum sensing and mac for opportunistic spectrum," presented at the Wireless Communications and Networking Conference Budapest, Apr. 2009.

[8] S. Stotas and A. Nallanathan. "On the throughput maximization of spectrum sharing cognitive radio networks," IEEE GLOBECOM'10, Miami, Dec. 2010. 